\title{
Dos perspectivas de una misma meta: pertinencia y nivel de logro de las competencias universitarias
}

\author{
María Luisa Belmonte \\ Mari Paz García-Sanz \\ Begoña Galián \\ Universidad de Murcia. España. \\ marialuisa.belmonte@um.es \\ maripaz@um.es \\ begona.g.n@um.es
}

Recibido: 30/4/2019

Aceptado: 16/1/2020

Publicado: 20/7/2020

\section{Resumen}

Tras las profundas modificaciones experimentadas por las instituciones educativas universitarias en los últimos años, se declara al estudiante como el mayor protagonista de su propio proceso formativo, lo que le promulga como agente activo de su aprendizaje. Dada la importancia que tiene la idoneidad de las competencias que los alumnos han de adquirir durante su paso por la universidad, así como el nivel de logro alcanzado en ellas, con el presente estudio se pretende evaluar y contrastar la percepción del profesorado y del alumnado en relación con la pertinencia de los conocimientos que conforman el grado en Pedagogía de la Universidad de Murcia, así como el nivel en que se han logrado, tanto de forma global como en función del curso en que se encuentran matriculados los estudiantes. A la luz de los resultados analizados se concluye que, en algunos casos, las opiniones de alumnos y docentes coinciden en cuanto a la pertinencia de las competencias, pero no en relación con el nivel de logro de las mismas. Únicamente los estudiantes de último curso, a nivel global, demuestran ideas concordantes con el profesorado en cuanto al grado competencial adquirido.

Palabras clave: educación; educación superior; alumnado; profesorado; modelo de formación competencial

Resum. Dues perspectives d'una mateixa fita: pertinència i nivell d'assoliment de les competències universitàries

Després de les profundes modificacions experimentades per les institucions educatives en els darrers anys, es declara l'estudiant com el protagonista més important del seu propi procés formatiu, amb la qual cosa és considerat un agent actiu del seu aprenentatge. Atesa la importància que té la idoneïtat de les competències que aquests alumnes han d'adquirir durant el seu pas per la universitat, així com el nivell d'assoliment aconseguit en aquestes, amb el present estudi es pretén avaluar i contrastar la percepció del professorat i de l'alumnat en relació amb la pertinència de les competències del grau en Pedagogia de la Universitat de Múrcia, així com el nivell en el qual s'han aconseguit, tant de forma global com segons del curs. En vista dels resultats analitzats, les opinions d'alumnes i docents coinci- 
deixen pel que fa a la pertinència de les competències, però no en relació amb el nivell d'assoliment d'aquestes. Únicament els estudiants d'últim curs demostren idees concordants pel que fa al grau competencial adquirit.

Paraules clau: educació; educació superior; alumnat; professorat; model de formació competencial

\section{Abstract. Two perspectives of the same goal: Relevance and level of achievement of university competences}

Following the profound changes higher education institutions have undergone in recent years, students are now considered the main protagonists of their own training and viewed as active agents in their learning process. In this new context, the suitability and level of competences students are expected to acquire have become an important aspect of their education. This study aims to evaluate and contrast teachers and students' perceptions of the acquisition and level of these competences in the Bachelor's Degree in Pedagogy at the University of Murcia, Spain, both globally and by academic year. The results show that students and teachers' opinions coincide regarding the relevance of the competences, but not in relation to their level of achievement. Only final year students agree on the level of competence acquired.

Keywords: education; higher education; students; teachers; competency-based education model

\begin{aligned} & \multicolumn{2}{c}{ Sumario } \\ & 1. Introducción 4. Resultados \\ & 2. Evaluando en el modelo 5. Discusión y conclusiones \\ & de formación por competencias Referencias bibliográficas \\ & 3. Metodología \end{aligned}

\section{Introducción}

El modelo educativo actual, basado en el aprendizaje de competencias por parte del estudiante, aboga por definir todos los elementos curriculares y organizativos en torno a las mismas (García-Sanz, 2011; López Ruiz, 2011; Martín del Pozo, Fernández-Lozano, González-Ballesteros y Juanas, 2013; Martín y Wolff, 2011; Torrado y Figuera, 2019).

Evaluar permite evidenciar la adquisición de la formación recibida, constatada en los resultados del aprendizaje, debiendo estar los métodos evaluativos alineados (Aneas, Rubio y Vilà, 2018). Por ello, la evaluación como elemento clave del currículum (Cowan, 2010), en continua interrelación con el resto, debe ir encaminada a poner de manifiesto el logro de competencias previamente determinadas (García-Sanz, 2014). Dado que las prácticas de enseñanza universitaria se están viendo renovadas, lo mismo se puede afirmar cuando se hace referencia a las prácticas evaluativas (Gilles, Detroz y Blais, 2011). 
Uno de los cambios que se ha venido desarrollando en las universidades europeas consiste en otorgar mayor participación a todos los agentes implicados, y en especial al alumnado, en los procesos de evaluación de adquisición de competencias (Hernández, 2012; Medina, Domínguez y Sánchez-Romero, 2013; Trevitt, Breman y Stocks, 2012), logrando con ello que las prácticas evaluativas tengan el potencial de involucrar a los estudiantes en la reflexión y que estos tomen un mayor control de su aprendizaje (Hodgson y Pang, 2012).

Aunque seamos conscientes de las limitaciones que ello conlleva (Braun, Woodley, Richardson y Leidner, 2012), sabemos que trabajar con los estudiantes sobre sus percepciones acerca del nivel de logro de sus competencias es un modo de involucrarle directamente en su propia evaluación y de potenciar la reflexión sobre la misma. Esta opinión, junto con la del profesorado, permite evidenciar si las calificaciones del alumnado son coherentes con el grado de desarrollo de las competencias (Goodman, Arbona y Domínguez, 2008), ya que, si bien esta relación debería darse en todas las ocasiones (Braun, Sheikh y Hannover, 2011), no siempre ocurre así (Lopes, Branco y JimenezAleixandre, 2011), puesto que prevalece una sobreestimación de los conocimientos adquiridos (Baartman y Ruijs, 2011).

Según González-López y López-Cámara (2010), el proceso evaluativo constituye uno de los pilares básicos en los que se ha de incidir con el fin de lograr el éxito en el proceso de enseñanza-aprendizaje, definido por el esquema de un modelo educativo que se guía por un alumnado como núcleo central del proceso, una verdadera autogestión del aprendizaje y una evaluación acorde con la consecución de competencias, entre otras.

Algunos modelos de enseñanza ciñen la evaluación a la adquisición de conocimientos específicos, pero en el proceso de evaluación por competencias dicha adquisición queda integrada en el nivel de dominio alcanzado en el desarrollo de la competencia, aplicando estrategias de evaluación encaminadas a conocer el grado de logro de las mismas.

En este estudio, de acuerdo con una de las cuestiones fundamentales para evaluar competencias que propone Fernández-March (2010), se trata de dar respuesta a cuáles son los niveles de desarrollo o dominio que tienen los estudiantes sobre las competencias.

\section{Evaluando en el modelo de formación por competencias}

La búsqueda de una delimitación conceptual del término competencia nos lleva a reflexionar sobre el carácter polisémico del mismo. De forma sintética, entendemos la competencia como la capacidad para seleccionar conocimientos, habilidades y actitudes para responder con éxito a una determinada situación.

En el ámbito universitario, las competencias que los estudiantes han de desarrollar, aparte de las establecidas por el Marco Español de Cualificaciones para la Educación Superior, son de dos tipos: genéricas o transversales y específicas. Las primeras son comunes a todas las titulaciones de una misma universidad, mientras que las segundas son específicas de cada titulación y de cada asignatura. 
En el contexto evaluativo, para hacer frente al modelo competencial, el profesorado ha de conocer y saber aplicar un sistema evaluativo variado $\mathrm{y}$ formativo, pero sobre todo continuo (Inda, Álvarez y Álvarez, 2008). Entendida de este modo, la evaluación se convierte en un proceso que activa, orienta y afecta (Huertas, 2009), ya que debe motivar al estudiante hacia el aprendizaje y orientarle hacia la mejora.

Dentro del modelo de formación en competencias, en el transcurso de la enseñanza y el aprendizaje, la evaluación es un proceso sistemático de recogida de información que va ligada a su interpretación, la cual se basa en una descripción valorativa que se comunica al estudiante con el fin de mejorar su aprendizaje, acreditando oficialmente el nivel de dominio de la competencia correspondiente (Villa y Poblete, 2011). De acuerdo con Castro (2011, p. 118):

[...] la evaluación de competencias se puede definir como un procedimiento en el que se requiere que el estudiante complete tareas o procesos en los que se demuestre su habilidad para aplicar conocimiento y destrezas o aplicar conocimientos en situaciones simuladas similares a la vida real a lo largo del tiempo.

Y según Brown (2015) está totalmente integrada en el proceso de enseñanza. Por ello, con un buen sistema de evaluación, los estudiantes no dudan de la calidad de su formación. Sin él, sin embargo, se sienten amenazados (McDonald, Boud, Francis y Gonzi, 2000).

La evaluación, por tanto, ha de ir encaminada a poner de manifiesto el logro de competencias previamente determinadas. Por ello, al llevar a cabo la planificación de las que deben desarrollar los estudiantes, no solo se debe hacer hincapié en cómo enseñarlas, sino también en cómo evaluarlas. De acuerdo con Segura (2009), la evaluación de los aprendizajes como parte inherente del currículo debe virar su modo de actuación, cambiando de una comprobación de resultados del alumno a la promoción de competencias, ya que, tal y como ocurre con la enseñanza universitaria en general, las prácticas de evaluación también se encuentran envueltas en un estado de cambio continuo (Gilles, Detroz y Blais, 2011), y esto se debe tomar en consideración.

Dicho proceso evaluativo es pues la herramienta que permite valorar el nivel de logro de las competencias que los estudiantes deben de adquirir en las diferentes titulaciones universitarias, actuando además de elemento regulador del aprendizaje (García-Sanz, 2014). Por todo ello la evaluación de competencias constituye una de las tareas más importantes en los procesos educativos universitarios (Cowan, 2010).

El modelo de formación en competencias implica un proceso de cambio que incide directamente en la visión del estudiante, puesto que fomenta el pensamiento crítico y su inteligencia práctica. Concretamente, este modelo supone una mayor transparencia de los perfiles profesionales en los programas de estudio y un especial énfasis en los resultados de aprendizaje, además del cambio de un enfoque educativo más orientado a la figura del alumno y a las respuestas a las demandas crecientes de una sociedad en un contexto de aprendizaje permanente. 
Entre los cambios que este modelo suscita se encuentra la transformación de la figura del profesor, quien pasa de asumir un rol de instructor al de docente con el papel de facilitador del aprendizaje (Ion y Cano, 2011). Se trata de un cambio de investigador y profesional dedicado casi exclusivamente a la transmisión de contenidos, a convertirse en un guía del alumno para la adquisición de las competencias requeridas. También el rol del estudiante evoluciona de asimilador de contenidos a elemento activo del proceso, colaborando en la elaboración de su propio currículo y desarrollo (Riverón, Mompié y Ramos, 2019). Perrenoud (2008, p. 8) estima que «no es una idea nueva que la evaluación puede ayudar al aprendizaje del alumno. Desde que existe la escuela, los pedagogos se rebelan contra las notas y quieren poner la evaluación al servicio del alumno, más que al del sistema». Manzanares y Sánchez Santamaría (2012) defienden que la evaluación tiene como fin recoger la complejidad de las competencias de acuerdo con el contexto profesional para el que se está formando al estudiante. Lógicamente, esta perspectiva integradora de las competencias debe reflejarse en las actividades de evaluación propuestas en cada materia. Según Sánchez Elvira, López González y Fernández Sánchez (2010), lo que ningún experto discute es que, partiendo de la base de que las competencias son conjuntos de saberes que quedan reflejados en el desempeño, ni su adquisición ni su evaluación pueden hacerse desde métodos tradicionales basados en el aprendizaje y el recuerdo o el reconocimiento de contenidos.

Esta participación del estudiante en la valoración de sus aprendizajes debe hacerse extensible a todos los elementos del currículo, mediante procedimientos de investigación que escuchen la voz del alumnado (Calvo y Susinos, 2010). De entre estos elementos, dentro del actual modelo educativo universitario destacan evidentemente las competencias. En relación con estas, el alumnado no únicamente ha de evaluar el nivel de logro de las que considera que ha adquirido, sino también su pertinencia, asegurando con ello la participación del mismo en la valoración de las competencias que se pretende desarrollar. Así, tras varios años de implantación del grado en Pedagogía en la Universidad de Murcia, se decide realizar esta investigación de carácter transversal, basada en la opinión de los dos agentes educativos principales.

El objetivo y el propósito de la misma es evaluar y contrastar la percepción del profesorado y del alumnado en relación con la pertinencia de las competencias incluidas en las guías docentes de las asignaturas que conforman dicho título, así como el grado en el que se han logrado dichas competencias, tanto de forma global como en función del curso en el que se encuentran matriculados los estudiantes.

\section{Metodología}

Esta investigación evaluativa cuantitativa, situada en la fase sumativa del ciclo de intervención educativa (García-Sanz, 2012), se contextualiza en la Universidad de Murcia, concretamente en el grado en Pedagogía de la Facultad de Educación de dicha universidad. 
El método de muestreo es el no probabilístico casual, puesto que en el estudio participaron los 168 estudiantes que se encontraban en el aula en el momento de efectuar la recogida de información, tras haber solicitado el permiso correspondiente al profesorado.

A nivel docente, participaron en la investigación 41 profesionales de la educación. En este caso se realizó un muestreo deliberado, ya que se invitó a intervenir en ella únicamente a los profesores que habían impartido docencia en el mencionado grado.

La distribución de la muestra en función del curso al que pertenecían alumnado y profesorado se presenta en la tabla 1 .

Tabla 1. Distribución muestral de profesores y alumnos en función del curso

\begin{tabular}{lccccc}
\hline & Primero & Segundo & Tercero & Cuarto & TOTAL \\
\hline Alumnos & 44 & 55 & 33 & 36 & 168 \\
\hline Profesores & 11 & 8 & 11 & 11 & 41 \\
\hline
\end{tabular}

Fuente: elaboración propia.

Para llevar a cabo la investigación se utilizaron cuestionarios ad hoc cuyos ítems se correspondían con cada una de las competencias contempladas en las guías docentes de las distintas asignaturas del grado (Facultad de Educación, 2019), con lo cual la validez del contenido de dichos instrumentos quedó garantizada. La escala utilizada tras cada competencia fue de cinco grados (1. Nada; 2. Poco; 3. Aceptable; 4. Bastante, y 5. Mucho) y los criterios de calidad para evaluar el grado de pertinencia de las competencias de las distintas asignaturas fueron: claridad, relevancia, evaluabilidad y exclusividad.

Respecto a la fiabilidad de los cuestionarios, estos demostraron poseer un alto grado de consistencia interna, puesto que en todos los casos el coeficiente alfa de Cronbach superaba la puntuación de 0,90.

Para analizar los datos se utilizó el paquete estadístico SPSS, versión 24. Para ello, se recurrió a la estadística descriptiva e inferencial paramétrica una vez analizadas las condiciones para poderla aplicar (Siegel, 1991), especialmente tras comprobar la distribución normal de la población y la homocedasticidad. En todos los casos se ha utilizado un nivel de significación estadística de alfa $=0,05$. Sin embargo, dado que la significación estadística no proporciona información sobre la fuerza de las correlaciones calculadas, también se ha considerado oportuno valorar la magnitud de la asociación entre variables a partir del denominado tamaño del efecto determinado por Cohen (1988), quien estimó que dicho valor debe compararse con un coeficiente de correlación mayor o igual a 0,3 . 


\section{Resultados}

Se presenta el nivel de pertinencia de las competencias de los datos procedentes de la aplicación de los cuestionarios en función de la opinión del alumnado y del profesorado, así como del grado en el que se han alcanzado dichas competencias, dependiendo del curso de los mismos y de los objetivos planteados.

\subsection{Percepción de la pertinencia otorgada a las competencias por profesorado y alumnado a nivel global}

En la tabla 2, tras aplicar el coeficiente de correlación de Spearman (Rho/ $\rho)$, se muestra la relación entre las opiniones de estudiantes y docentes relativas a la pertinencia de las competencias, tanto consideradas conjuntamente como diferenciando entre competencias transversales y específicas.

A nivel holístico existe una correlación positiva y moderada $\left(\rho_{G}=0,470\right)$ entre la percepción que posee el alumnado sobre la pertinencia de las competencias y la del profesorado, resultando además ser estadísticamente significativa $(p=0,012)$, con un tamaño del efecto superior al típico establecido por Cohen (1988).

Las opiniones de sendos colectivos se encuentran ligeramente correlacionadas positivamente, tanto a nivel transversal $\left(\rho_{\mathrm{T}}=0,299\right)$ como específico $\left(\rho_{\mathrm{E}}=0,367\right)$, aunque esta vez sin reflejar significación estadística $(p=0,122$; $p=0,055)$.

Tabla 2. Correlación entre la percepción del profesorado y del alumnado sobre la pertinencia de las competencias en el grado de Pedagogía

\begin{tabular}{lcc}
\hline Tipo de competencia & Rho & Sig. bilateral \\
\hline Global & 0,470 & 0,012 \\
Transversales & 0,299 & 0,122 \\
\hline Específicas & 0,367 & 0,055 \\
\hline
\end{tabular}

Fuente: elaboración propia.

\subsection{Percepción de la pertinencia otorgada a las competencias por profesorado y alumnado en función del curso de que se trate}

La tabla 3 muestra que, a nivel general, existe una correlación baja y negativa $\left(\rho_{\mathrm{G}}=-0,183\right)$, no significativa $(p=0,613)$ entre la percepción que tiene el alumnado y el profesorado sobre la pertinencia de las competencias incluidas en las asignaturas del primer curso de grado en Pedagogía. Esto ocurre tanto a nivel general como en lo que respecta a las competencias transversales $\left(\rho_{\mathrm{T}}=\right.$ $-0,340 ; p=0,336)$ y específicas $\left(\rho_{\mathrm{E}}=-0,067 ; p=0,854\right)$. 
Tabla 3. Correlación entre la percepción del profesorado y del alumnado sobre la pertinencia de las competencias del primer curso

\begin{tabular}{lcc}
\hline Tipo de competencia & Rho & Sig. bilateral \\
\hline Global & $-0,183$ & 0,613 \\
Transversal & $-0,340$ & 0,336 \\
\hline Específica & $-0,067$ & 0,854 \\
\hline
\end{tabular}

Fuente: elaboración propia.

En la tabla 4 se puede contrastar que, en el segundo curso, tanto a nivel global $\left(\rho_{\mathrm{G}}=0,786\right)$ como en las competencias transversales $\left(\rho_{\mathrm{T}}=0,786\right)$, existe una correlación medio-alta y significativa ( $p=0,036$ para ambos casos), arrojando un tamaño del efecto más que suficiente, por lo que alumnos y docentes coinciden a la hora de valorar la pertinencia de este tipo de competencias. En el caso de las competencias específicas de las asignaturas del segundo curso la correlación puede entenderse como moderada $\left(\rho_{E}=0,500\right)$, no siendo significativa $(p=0,253)$.

Tabla 4. Correlación entre la percepción del profesorado y del alumnado sobre la pertinencia de las competencias del segundo curso

\begin{tabular}{lcc}
\hline Tipo de competencia & Rho & Sig. bilateral \\
\hline Global & 0,786 & 0,036 \\
\hline Transversal & 0,786 & 0,036 \\
\hline Específica & 0,500 & 0,253 \\
\hline
\end{tabular}

Fuente: elaboración propia.

Tal y como se presenta en la tabla 5 , en el tercer curso y a nivel global existe una correlación baja y positiva $\left(\rho_{\mathrm{G}}=0,290\right)$ que resulta no ser significativa $(p=0,577)$. Lo mismo ocurre en cuanto a las competencias transversales $\left(\rho_{\mathrm{T}}=0,261 ; p=0,618\right)$ y específicas $\left(\rho_{\mathrm{E}}=0,203 ; p=0,700\right)$, por lo que, al igual que en el primer curso, no existe una relación significativa entre la opinión de los alumnos y la de los docentes acerca de la pertinencia de las competencias incluidas en las guías docentes de las asignaturas del mencionado curso.

Tabla 5. Correlación entre la percepción del profesorado y del alumnado sobre la pertinencia de las competencias del tercer curso

\begin{tabular}{lcc}
\hline Tipo de competencia & Rho & Sig. bilateral \\
\hline Global & 0,290 & 0,577 \\
Transversal & 0,261 & 0,618 \\
Específica & 0,203 & 0,700 \\
\hline
\end{tabular}

Fuente: elaboración propia. 
Por último, en la tabla 6 se observa que en el cuarto curso existe una correlación muy alta entre sendos colectivos, tanto a nivel competencial general $\left(\rho_{\mathrm{G}}\right.$ $=0,900)$ como en lo que respecta a las competencias transversales $\left(\rho_{\mathrm{T}}=0,900\right)$, siendo además significativa ( $p=0,037$ en ambos casos) y con un tamaño del efecto considerablemente superior al típico establecido por Cohen (1988). Es decir, alumnos y docentes, al igual que ocurre en segundo, coinciden en cuanto a la valoración de la pertinencia de las competencias de este tipo. En cambio, contrariamente, en lo que concierne a las de tipo específico $\left(\rho_{\mathrm{E}}=0,100\right)$, la correlación es muy baja y no significativa $(p=0,873)$.

Tabla 6. Correlación entre la percepción del profesorado y del alumnado sobre la pertinencia de las competencias del cuarto curso

\begin{tabular}{lcc}
\hline Tipo de competencia & Rho & Sig. bilateral \\
\hline Global & 0,900 & 0,037 \\
\hline Transversal & 0,900 & 0,037 \\
\hline Específica & 0,100 & 0,873 \\
\hline
\end{tabular}

Fuente: elaboración propia.

\subsection{Percepción del nivel de logro otorgado a las competencias por el profesorado y el alumnado a nivel global}

En la tabla 7 se muestran los coeficientes de correlación (Rho/ $\rho$ ), correspondientes a la relación existente entre la valoración que realizan los alumnos y los profesores sobre el logro de las competencias (a nivel global y diferenciando entre transversales y específicas) incluidas en las asignaturas del grado de Pedagogía.

Considerando conjuntamente las competencias transversales y específicas, existe una relación baja, positiva $\left(\rho_{\mathrm{G}}=0,160\right)$ y no significativa $(p=0,417)$ entre la valoración que los alumnos y los docentes otorgan al logro de las competencias. Lo mismo ocurre diferenciando entre competencias transversales $\left(\rho_{\mathrm{T}}=0,272 ; p=0,161\right)$ y específicas $\left(\rho_{\mathrm{E}}=0,238 ; p=0,223\right)$.

Tabla 7. Correlación entre la percepción del profesorado y del alumnado sobre el nivel de logro de las competencias en el grado en Pedagogía

\begin{tabular}{lcc}
\hline Tipo de competencia & Rho & Sig. bilateral \\
\hline Global & 0,160 & 0,417 \\
\hline Transversal & 0,272 & 0,161 \\
\hline Específica & 0,238 & 0,223 \\
\hline
\end{tabular}

Fuente: elaboración propia. 


\subsection{Percepción del nivel de logro otorgado a las competencias por el profesorado $y$ el alumnado en función del curso de los mismos}

Con respecto al primer curso, se observa en la tabla 8 que, consideradas las competencias conjuntamente, existe una baja correlación inversa entre docentes y estudiantes en lo que respecta al nivel de logro competencial adquirido por estos $\left(\rho_{G}=-0,224\right)$, sin que dicha correlación resulte ser significativa $(p=0,533)$. Con respecto a las competencias transversales, existe una leve correlación inversa $\left(\rho_{\mathrm{T}}=-0,141\right)$ y no significativa $(p=0,698)$, ocurriendo algo similar con las competencias específicas $\left(\rho_{\mathrm{E}}=-0,037 ; p=0,920\right)$.

Tabla 8. Correlación entre la percepción del profesorado y del alumnado sobre el nivel de logro de las competencias del primer curso

\begin{tabular}{lcc}
\hline Tipo de competencia & Rho & Sig. bilateral \\
\hline General & $-0,224$ & 0,533 \\
Transversal & $-0,141$ & 0,698 \\
\hline Específica & $-0,037$ & 0,920 \\
\hline
\end{tabular}

Fuente: elaboración propia.

En relación con el segundo curso, la tabla 9 señala que, a nivel general, se obtiene una correlación moderada y positiva entre la opinión de sendos colectivos $\left(\rho_{\mathrm{G}}=0,445\right)$, sin que resulte ser significativa $(p=0,317)$. En cuanto a las competencias transversales, a pesar de que el coeficiente de correlación de Spearman obtiene un valor medio-alto $\left(\rho_{\mathrm{T}}=0,741\right)$, la relación tampoco es significativa $(p=0,057)$. Asimismo, la percepción de logro de los dos colectivos sobre las competencias específicas de las asignaturas del segundo curso presenta una baja relación positiva $\left(\rho_{\mathrm{E}}=0,118\right)$, sin ser significativa $(p=0,801)$.

Tabla 9. Correlación entre la percepción del profesorado y del alumnado sobre el nivel de logro de las competencias del segundo curso

\begin{tabular}{lcl}
\hline Tipo de competencia & Rho & Sig. c \\
\hline General & 0,445 & 0,317 \\
Transversal & 0,741 & 0,057 \\
\hline Específica & 0,118 & 0,801 \\
\hline
\end{tabular}

Fuente: elaboración propia.

En la tabla 10 se puede comprobar que las opiniones sobre el logro de las competencias consideradas conjuntamente, según el alumnado y el profesorado, tienen una baja, positiva y no significativa relación con el tercer curso $\left(\rho_{\mathrm{G}}=0,257 ; p=0,623\right)$. Más concretamente, la percepción de logro de las competencias transversales $\left(\rho_{\mathrm{T}}=0,500\right)$ se correlaciona de forma moderada y positiva, sin llegar a ser significativa $(p=0,313)$, y las competencias específicas $\left(\rho_{E}=0,232\right)$ presentan una correlación baja, positiva $y$, al igual que en las anteriores, no significativa $(p=0,658)$. 
Tabla 10. Correlación entre la percepción del profesorado y del alumnado sobre el nivel de logro de las competencias del tercer curso

\begin{tabular}{lcc}
\hline Tipo de competencia & Rho & Sig. bilateral \\
\hline General & 0,257 & 0,623 \\
Transversal & 0,500 & 0,313 \\
Específica & 0,232 & 0,658 \\
\hline
\end{tabular}

Fuente: elaboración propia.

En la tabla 11 se muestra la relación existente entre las opiniones de los alumnos y de los docentes con respecto al logro de las competencias en el cuarto curso. Como se aprecia, a nivel general existe una alta correlación positiva $\left(\rho_{\mathrm{G}}=0,900\right)$ que resulta ser significativa $(p=0,037)$ y con un alto tamaño del efecto. Esto implica la existencia de una relación entre las opiniones de ambos colectivos con respecto al logro de las competencias de las asignaturas del último curso de la titulación. En cuanto a las competencias transversales, se halla una relación medio-alta y positiva $\left(\rho_{\mathrm{T}}=0,700\right)$, aunque no significativa $(p=0,188)$. Igualmente, la relación entre las opiniones de los docentes y los estudiantes con respecto al nivel de logro de las competencias específicas del cuarto curso es moderada y positiva $\left(\rho_{\mathrm{E}}=0,564\right)$, aunque tampoco significativa $(p=0,322)$.

Tabla 11. Correlación entre la percepción del profesorado y del alumnado sobre el nivel de logro de las competencias del cuarto curso

\begin{tabular}{lcc}
\hline Tipo de competencia & Rho & Sig. bilateral \\
\hline General & 0,900 & 0,037 \\
\hline Transversal & 0,700 & 0,188 \\
\hline Específica & 0,564 & 0,322 \\
\hline
\end{tabular}

Fuente: elaboración propia.

\section{Discusión y conclusiones}

Tal y como demuestran diversos estudios, la evaluación es uno de los principales recursos que orientan al estudiante sobre cómo aprender (Nijhuis, 2010; Tobón, 2010). La evaluación de competencias y por competencias es un proceso de retroalimentación y certificación de los aprendizajes (Zabalza, 2003). Esto tiene como consecuencia importantes cambios en la evaluación tradicional, ya que en este enfoque de valoración los estudiantes deben ser muy conscientes del para qué, para quién y cómo es la evaluación, o por qué es tan necesaria para contribuir a formar profesionales idóneos. Es así como la evaluación debe plantearse mediante realidades que impliquen motivación, curiosidad y reto (Tejada, 2011), ya que el método evaluativo tradicional, instaurado en las universidades españolas, versa en torno al trabajo y al esfuerzo del profesor, lo que propicia una actitud estudiantil pasiva y limita su función a 
recibir información para plasmarla en un examen final, normalmente muy teórico (Calvo y Mingorance, 2010).

En este sentido, se requiere una educación que forme ciudadanos competentes en un medio cada vez más exigente que evoluciona con criterios de calidad, y que demande mayor capacidad de adaptación a las nuevas circunstancias (Díaz, 2017). Por ello pensar en evaluación es también considerar la nueva educación que se desea (Rigo y Donolo, 2016).

Dado que las instituciones vienen experimentando en los últimos años grandes y profundas modificaciones para dar respuesta a un contexto cada vez más complejo e incierto, autores como Ojeda (2015) defienden que en estos tiempos en los que la universidad es uno de los puntos álgidos del centro del crecimiento, desarrollo y mejora de la sociedad, su papel es crítico y crucial. Por ello, aunque «acercar la universidad a la vida ha sido un reclamo ancestral, en estos tiempos se hace un imperativo» (Pimienta, 2011, p. 77), sin que se llegue a producir el mercantilismo de la educación. Esta pedagogía enfocada hacia la adquisición de competencias hace ya tiempo que no basa sus actuaciones en lo que el estudiante deberá haber interiorizado al concluir una materia o un ciclo, sino en las acciones que tendrá que ser capaz de efectuar una vez superado un periodo de aprendizaje (De Miguel, 2005), por ello resulta más que conveniente que se busquen procedimientos eficaces para evaluar los programas educativos desde el logro del perfil de egreso (Carrera, Lara y Madrigal, 2019).

En la presente investigación se considera la opinión tanto del alumnado como del profesorado sobre la pertinencia de las competencias incluidas en las guías docentes de las asignaturas que conforman el título de grado en Pedagogía, así como el nivel en el que afirman que se han logrado dichas competencias, tanto de forma global como en función del curso en el que se encuentran matriculados los estudiantes.

En cuanto a la percepción que poseen alumnado y profesorado sobre la pertinencia de las competencias incluidas en las guías docentes de las asignaturas del grado en Pedagogía, a nivel general se halla una correlación positiva y moderada, estadísticamente significativa, que arroja un tamaño del efecto superior al típico establecido por Cohen.

En un nivel de concreción mayor, en el segundo curso, la opinión de los dos agentes educativos sobre las competencias, tanto a nivel global como haciendo referencia a las de carácter transversal, existe una correlación medioalta y significativa, con un tamaño del efecto más que suficiente para ambos casos, por lo que estudiantes y docentes coinciden a la hora de valorar la pertinencia de dichas competencias. También en el cuarto curso existe una correlación muy alta y significativa y con un tamaño del efecto considerablemente superior al típico, entre las opiniones de sendos colectivos, tanto a nivel competencial general como, de nuevo, en lo que respecta a las competencias transversales. Es decir, alumnos y profesores coinciden en cuanto a la valoración de la pertinencia de las competencias de este tipo. Estas similitudes entre percepciones de competencias transversales pueden deberse al carácter genérico de las mismas (Macías, Rodríguez Sánchez y Aguilera, 2017). 
Por último, en cuanto a la percepción de estudiantes y docentes sobre el grado en el que se han logrado dichas competencias, únicamente en el cuarto curso se aprecia, a nivel general, una elevada correlación positiva significativa, con un tamaño del efecto muy alto. Esto implica la existencia de una relación entre las opiniones de los alumnos de mayor edad y del profesorado con respecto al logro de las competencias de las asignaturas del último curso de la titulación. En otras investigaciones realizadas en la Universidad de Murcia los estudiantes de Medicina valoran el logro de competencias en la asignatura de Traumatología con puntuaciones superiores a las concedidas por el profesorado (García-Sanz et al., 2017), lo que conlleva también bajos coeficientes de correlación. La misma coyuntura se da en los resultados obtenidos por GarcíaSanz et al. (2009), al evaluar años anteriores las competencias adquiridas por el alumnado del primer curso del grado en Pedagogía, quienes encontraron diferencias significativas en lo que respecta al nivel de logro de las competencias (tanto específicas como transversales) entre las puntuaciones otorgadas por el alumnado y el profesorado, a favor de los primeros.

Todas estas conclusiones extraídas, envueltas en el contexto de la creciente preocupación por aspectos relativos al crecimiento de la calidad pedagógica (Bozu y Manolescu, 2010), demuestran la necesidad de realizar una revisión de las guías docentes de las asignaturas (Herrero, González y Martín, 2015), por lo que, en primer lugar, se propone que este sea el siguiente paso como prospectiva de la investigación mostrada. Asimismo, en segundo lugar, se sugiere revisar la forma de enseñar y de evaluar las competencias que ha de desarrollar el alumnado. De acuerdo con Díaz (2017), el progreso de la calidad y el aprendizaje de los estudiantes debe asumir en años venideros un papel pedagógicamente más relevante dentro de un contexto mundial.

\section{Referencias bibliográficas}

Aneas, A.; Rubio, M.J. y Vilà, R. (2018). Portafolios digital y evaluación de las competencias transversales en las prácticas externas del grado de Pedagogía de la Universidad de Barcelona. Educar, 54(2), 283-301. $<$ https://doi.org/10.5565/rev/educar.878>

BaARTMAn, L. y Ruijs, L. (2011). Comparing students' perceived and actual competence in higher vocational education. Assessment \& Evaluation in Higher Education, 36(4), 385-398.

Bozu, Z. y Manolescu, M. (2010). El espacio europeo de educación superior y el profesorado universitario novel: Un estudio cualitativo. Bordón, 62(4), 51-63.

Braun, E.; Sheikh, H. y Hannover, B. (2011). Self-rated competences and future vocational success: A longitudinal study. Assessment \& Evaluation in Higher Education, 36(4), 417-427.

Braun, E.; Woodley, A.; Richardson, J. y Leidner, B. (2012). Self-rated competences questionnaires from a design perspective. Educational Research Review, 7(1), $1-18$.

Brown, S. (2015). La evaluación auténtica: El uso de la evaluación para ayudar a los estudiantes a aprender. Revista Electrónica de Investigación y Evaluación Educativa, 21(2), 1-10. 
Calvo, A. y Mingorance, C. (2010). Evaluación continua de conocimientos vs de competencias: Resultados de la aplicación de dos métodos valorativos diferentes. Revista de Investigación Educativa, 28(2), 361-383.

Calvo, A. y Susinos, T. (2010). Prácticas de investigación que escuchan la voz del alumnado: Mejorar la universidad indagando la experiencia. Revista de Currículum y Formación del Profesorado, 14(3), 75-88.

Carrera, C.; Lara, Y.I. y Madrigal, J. (2019). Evaluación del logro del perfil de egreso: Un estudio de caso. RECIE: Revista Electrónica Cientifica de Investigación Educativa, 4(2), 1019-1028. Recuperado de <http://www.rediech.org/ojs/2017/ index.php/recie/article/view/317>.

CAstro, M. (2011). ¿Qué sabemos de la medida de las competencias?: Características y problemas psicométricos en la evaluación de competencias. Bordón, 63(1), 109-123.

Cohen, J. (1988). Statistical Power Analysis for the Behavioral Sciences. 2. a ed. Nueva York: Academic Press.

CowAN, J. (2010). Developing the ability for making evaluative judgements. Teaching in Higher Education, 15(3), 323-334.

Díaz, A.M. (2017). Evaluación del programa Todos a Aprender en la enseñanza de lenguaje y matemáticas en el municipio de Puerto Escondido, Córdoba. Panorama, 10(19), 46-59.

<http://dx.doi.org/10.15765/pnrm.v10i19.929>

Facultad de Educación (2019). Guías docentes curso 2019/2020 (Grado en Pedagogia). Recuperado de <https://www.um.es/web/educacion/contenido/estudios/ grados/pedagogia/2019-20/guias $>$.

Fernández March, A. (2010). Cambio de la cultura de evaluación educativa no solo de métodos. Comunicación presentada en el Seminario Internacional de trabajo sobre la evaluación a través de rúbricas. UPV, San Sebastián Donostia.

GARCíA-SANZ, M.P. (2011). Innovaciones orientadas al EEES: Las competencias y su evaluación como elementos de planificación en el marco del EEES. En J.J. MAQUILÓN (coord.). La formación del profesorado en el siglo XXI: Propuestas ante los cambios económicos, sociales y culturales (pp. 63-80). Murcia: Editum.

- (2012). Fundamentos teóricos y metodológicos de la evaluación de programas. Murcia: DM.

- (2014). La evaluación de competencias en Educación Superior mediante rúbricas: Un caso práctico. Revista Electrónica Interuniversitaria de Formación del Profesorado, 17(1), 87-106.

<http://dx.doi.org/10.6018/reifop.17.1.198861>

García-Sanz, M.P.; García-Estañ, J.; Martínez-Martínez, F.; Pérez-Bernabeu, A. y Santonja, F. (2017). Evaluación de competencias prácticas clínicas por el alumnado y el profesorado a través del portafolios. Revista Complutense de Educación, 28(3), 737-754.

García-Sanz. M.P.; García-Sánchez, F.A.; Maquilón, J.J.; Vallejo, M.; Dorado, M.A.; Egea, D.A.; ... Ortega, P. (2009). Adaptación al EEES del primer curso de la titulación de Pedagogía. En P. Arnáiz, L. Hernández-Abenza y M.P. GarCÍA-SANZ (coords.). Experiencias de innovación educativa en la Universidad de Murcia (2008) (pp. 257-280). Murcia: Editum.

Gilles, J.L.; Detroz, P. y Blais, J.G. (2011). An international online survey of the practices and perceptions of higher education professors with respect to the assessment of learning in the classroom. Assessment \& Evaluation in Higher Education, 36(6), 719-733. 
González lópez, I. y López Cámara, A.B. (2010). Sentando las bases para la construcción de un modelo de evaluación a las competencias docentes del profesorado universitario. Revista de Investigación Educativa, 28(2), 403-423.

Goodman, G.; Arbona, C. y Domínguez de Rameriz, R. (2008). High-Stakes, Minimum-Competency Exams: How Competent Are They for Evaluating Teacher Competence? Journal of Teacher Education, 59(1), 24-39.

Hernández, R. (2012). Does continuous assessment in higher education support student learning? Higher Education, 64(4), 489-502.

Herrero, R.; González, I. y Martín, V. (2015). Formación centrada en competencias estudiantiles en Educación Superior. Revista de Ciencias Sociales, 21(4), 461-478.

Hodgson, P. y PAng, M. (2012). Effective formative e-assessment of student learning: A study on a statistics course. Assessment \& Evaluation in Higher Education, 37(2), $215-225$.

Huertas, J.A. (2009). Aprender a fijarse metas: Nuevos estilos motivacionales. En J.I. Pozo y M.P. PÉrez (coords.). Psicología del aprendizaje universitario: La formación en competencias (pp. 164-181). Madrid: Morata.

Inda, M.; Álvarez González, S. y Álvarez Rubio, R. (2008). Métodos de evaluación en la enseñanza superior. Revista de Investigación Educativa, 26(2), 539-552.

Ion, G. y Cano, E. (2011). El proceso de implementación de la evaluación por competencias en la Educación Superior: Un estudio del rol de los cargos académicos. Revista de Investigación en Educación, 9(2), 246-258.

Lopes, J.B.; Branco, J. y Jimenez-Aleixandre, M.P. (2011). 'Learning experience' provided by science teaching practice in a classroom and the development of students' competences. Research in Science Education, 41(5), 787-809. <https://doi.org/10.1007/s11165-010-9190-5>

López Ruiz, J.I. (2011). Un giro copernicano en la enseñanza universitaria: Formación por competencias. Revista de Educación, 356, 279-301.

Macías, E.; Rodríguez-Sánchez, M. y Aguilera, J.L. (2017). La cuestión universitaria: Adquisición de competencias transversales a través de tutoría en la universidad. Madrid: Universidad Complutense de Madrid.

Manzanares, M.A. y Sánchez Santamaría, J. (2012). La dimensión pedagógica de la evaluación por competencias y la promoción del desarrollo profesional en el estudiante universitario. Revista Iberoamericana de Evaluación Educativa, 5(1), 186-202.

Martín, P. y Wolff, L. (2011). La implantación de los nuevos grados: Propuestas de mejora. Revista de Educación, 356, 703-715.

Martín del Pozo, R.; Fernández Lozano, P.; González Ballesteros, M. y JuaNAS, A. de (2013). El dominio de los contenidos escolares: Competencia profesional y formación inicial de maestros. Revista de Educación, 360, 363387.

Mcdonald, R.; Boud, D.; Francis, J. y Gonzci, A. (2000). Nuevas perspectivas sobre la evaluación. Boletín Cinterfor, 149, 41-72.

Medina, A.; Domínguez, M.C. y Sánchez Romero, C. (2013). Evaluación de las competencias de los estudiantes: Modelos y técnicas para la valoración. Revista de Investigación Educativa, 31(1), 239-255.

Miguel, M. de (2005). Cambio de paradigma metodológico en la Educación Superior: Exigencias que conlleva. Cuadernos de Integración Europea, 2, 16-27.

Nijhuis, J. (2010). Redesigning a learning and assessment environment: The influence on students' perceptions of assessment demands and their learning strategies. Studies in Educational Evaluation, 32(3), 223-242. 
OJEDA, J.A. (2015). La crisis de la Universidad y su transformación: La colaboración y el trabajo en red. Qurriculum: Revista de Teoría, Investigación y Práctica Educati$v a, 28,190-211$.

Perrenoud, P. (2008). La evaluación de los alumnos: De la producción de la excelencia a la regulación de los aprendizajes. Buenos Aires: Colihue.

Pimienta, J.H. (2011). Secuencias didácticas: Aprendizaje y evaluación de competencias en educación superior. Bordón, 63(1), 77-92.

Rigo, D.Y. y Donolo, D. (2016). Evaluación... Más de lo mismo desafiando formatos y modalidades sin libreto. Panorama, 10(19), 62-75. $<$ http://dx.doi.org/10.15765/pnrm.v10i19.829>

Riverón, Y.; Mompié, A.D. y Ramos, A.E. (2019). El proceso de evaluación como contextualizador de la autorregulación del aprendizaje. Roca: Revista CientíficoEducacional de la Provincia Granma, 15(1), 186-198.

Sánchez-Elvira, A.; López-González, M.A. y Fernández-Sánchez, M. (2010). Análisis de las competencias genéricas en los nuevos títulos de grado del EEES en las universidades españolas. Revista de Docencia Universitaria, 8(1), 35-73.

SEGURA, M.A. (2009). La evaluación de los aprendizajes basada en el desempeño por competencias. Actualidades Investigativas en Educación, 9(2), 1-25.

Siegel, S. (1991). Estadística no paramétrica para ciencias de la conducta. México: Trillas.

TejadA, J. (2011). La evaluación de las competencias en contextos no formales: Dispositivos e instrumentos de evaluación. Revista de Educación, 354, 731-745.

Тово́n, S. (2010). Formación integral y competencias: Pensamiento complejo, currículo, didáctica y evaluación. Bogotá: ECOE Editores.

Torrado, M. y Figuera, P. (2019). Estudio longitudinal del proceso de abandono y reingreso de estudiantes de Ciencias Sociales: El caso de Administración y Dirección de Empresas. Educar, 55(2), 401-417. <https://doi.org/10.5565/rev/educar.1022>

Trevitt, C.; Breman, E. y Stocks, C. (20I2). Assessment and learning: Is it time to rethink student activities and academic roles. Revista de Investigación Educativa, 30(2), 253-269.

Villa, A. y Poblete, M. (2011). Evaluación de competencias genéricas: Principios, oportunidades y limitaciones. Bordón, 63(1), 147-170.

Zabalza, M.A. (2003). Competencias docentes del profesorado universitario: Calidad y desarrollo profesional. Madrid: Narcea. 